Original Article (short paper)

\title{
Exercises with action observation contribute to upper limb recovery in chronic stroke patients: a controlled clinical trial
}

\author{
Angela Cristina de Lima ${ }^{1,2}$ (D) , Gustavo Christofoletti ${ }^{2,3}$ (iD) \\ ${ }^{\prime}$ Universidade Federal da Grande Dourados, Hospital Universitário, Dourados, MS, Brasil. \\ ${ }^{2}$ Universidade Federal de Mato Grosso do Sul, Programa de Pós-graduação em Saúde e \\ Desenvolvimento da Região Centro-Oeste, Campo Grande, MS, Brasil. \\ ${ }^{3}$ Universidade Federal de Mato Grosso do Sul, Programa de Pós-Graduação em Ciências do \\ Movimento, Campo Grande, MS, Brasil.
}

\begin{abstract}
Aims: To investigate the effects of an exercise program with action observation versus conventional physical therapy on upper limb functionality in chronic stroke subjects. Methods: In this controlled clinical trial, thirtyfive stroke patients were divided into two groups, experimental group, comprising eighteen patients that received an exercise program with action observation; and a control group, comprising seventeen patients that received conventional exercise program. Functional recovery was assessed with the Fugl-Meyer Scale, manual dexterity was assessed with the Box and Blocks test, and the functional use of the affected upper limb was assessed with the Reach scale. Evaluations occurred at baseline, after three and six months of intervention. Statistical analyses were performed with the Repeated Measures Analysis of Variance and the Friedman test, under a 5\% significance. Results: Both interventions provided benefits to chronic stroke patients. Exercise program with action observation presented better results on motor recovery $(\mathrm{p}<0.001)$ and functional use of the affected limb $(\mathrm{p}<0.001)$ when compared with conventional therapy. Both treatments improved the manual dexterity of the participants $(p=0.002)$, but in a similar way $(p=0.461)$. Conclusion: A six-month exercise program with action observation provided benefits on functional recovery and functional use of an affected upper limb in chronic stroke patients. Exercises with action observation demonstrated the potential for improving affected upper limb in chronic stroke patients.
\end{abstract}

Keywords: stroke, exercise therapy, clinical trial, hemiplegia.

\section{Introduction}

Recovery of the affected upper limb is one of the great challenges in the rehabilitation of stroke patients. Approximately $60 \%$ of severely affected individuals do not present manual dexterity six months after the stroke ${ }^{1}$. The functional deficits of the upper limb affect the ability for self-care, contribute to low perceived quality of life and higher healthcare services costs ${ }^{2,3}$.

Exercise programs should start early, be intensive and developed with the active participation of patients to promote motor learning and minimize functional deficits ${ }^{4}$. Action observation training (AO) is an alternative treatment in which the individual observes an action and then imitates the task ${ }^{5}$.

AO stimulates the mirror neuron system, a special type of neurons activated by the execution and observation of action ${ }^{6}$. Initially studied in monkeys, the mirror neuron system is associated with the premotor cortex, supplementary motor area, primary somatosensory cortex, and inferior parietal cortex. By its connections with neurocognitive processing, exercises programs that stimulate the mirror neuron system may promote important benefits to stroke patients ${ }^{6,7}$.

During AO there is an activation of several cortical areas. An internal representation of the action can potentiate motor learning and functional recovery ${ }^{8,9}$. AO uses movements guided by external stimuli in which visual attention recruits the cerebellar-thalamic-cortical circuit ${ }^{10}$. This circuit is involved in neural integration during the initial stages of motor learning. In this matter, Wright and collegues ${ }^{11}$ showed that directed attention facilitates corticospinal excitability of the brain.

Previous studies using AO showed positive results in the recovery of the affected upper limb in stroke $\mathrm{e}^{12-17}$. There were improvements in functionality, on the ability to perform activities of daily living and on manual dexterity. Nevertheless, there are still few studies comparing the effects of $\mathrm{AO}$ versus conventional therapy (exercise without $\mathrm{AO}$ ) aiming to see how effective $\mathrm{AO}$ is in relation to exercise programs already consecrated.

Thus, in the present study, we investigated the benefits of $\mathrm{AO}$ in comparison to conventional physical therapy on upper limb functional recovery, manual dexterity and 
everyday use of the affected upper limb in individuals with stroke. We hypothesized that AO would present better outcomes to patients with stroke than a conventional exercise program.

\section{Methods}

\section{Trial design}

This is a controlled clinical trial comparing the effects of $\mathrm{AO}$ versus conventional exercise program (without AO) in chronic stroke patients. The research project was approved by the research ethics committee of the University Center of Grande Dourados - Unigran (CAAE: 31574214400005159, Number: 763.719), and it was prospectively registered in the Brazilian Registry of Clinical Trials (Protocol no. RBR-26Q4Z9). An informed consent form was signed by all the participants.

\section{Eligibility criteria and sample size calculation}

Forty participants diagnosed with a single stroke were eligible for this study. Inclusion criteria involved hemiparetic patients with brachiofacial predominance resulting from a stroke, with preserved visual acuity and absence of anosognosia. Exclusion criteria involved patients in the acute phase of the stroke ( $<3$ months), those with apraxia, agnosia, in use of medications to treat spasticity, subjects with cognitive decline or other neurologic disorder.
The sample size of this study was estimated assuming a power of $80 \%$, with a $5 \%$ type I error and an effect size of 0.779 . The effect size was based on the previous study developed by Peg and collegues ${ }^{18}$. With this methodological design, a minimum of thirty-five subjects showed to be appropriate to control type 1 and 2 errors.

\section{Participants}

Participants were divided into two groups: Experimental and control group. Both groups were similar regarding participants' age, time since diagnosis, type of stroke and affected cerebral hemisphere. All participants were right-handed and were classified from 1-3 according to the Ashworth Scale. Figure 1 details the flow of participant selection and monitoring during the study. Table 1 shows the sociodemographic and clinical conditions of the participants.

\section{Therapeutic protocol}

The experimental group received an exercise program with AO by watching videos of functional movements while imitating them. Thirty-five videos with upper limb exercises were prepared for the intervention, from a third-person perspective, as though the patient were looking in a mirror. All the movements were performed bilaterally so that regardless of the affected side the patient had the correct perspective to perform the exercise. The exercises involved bimanual movements and included selective movements of the shoulder, elbow, wrist, and finger

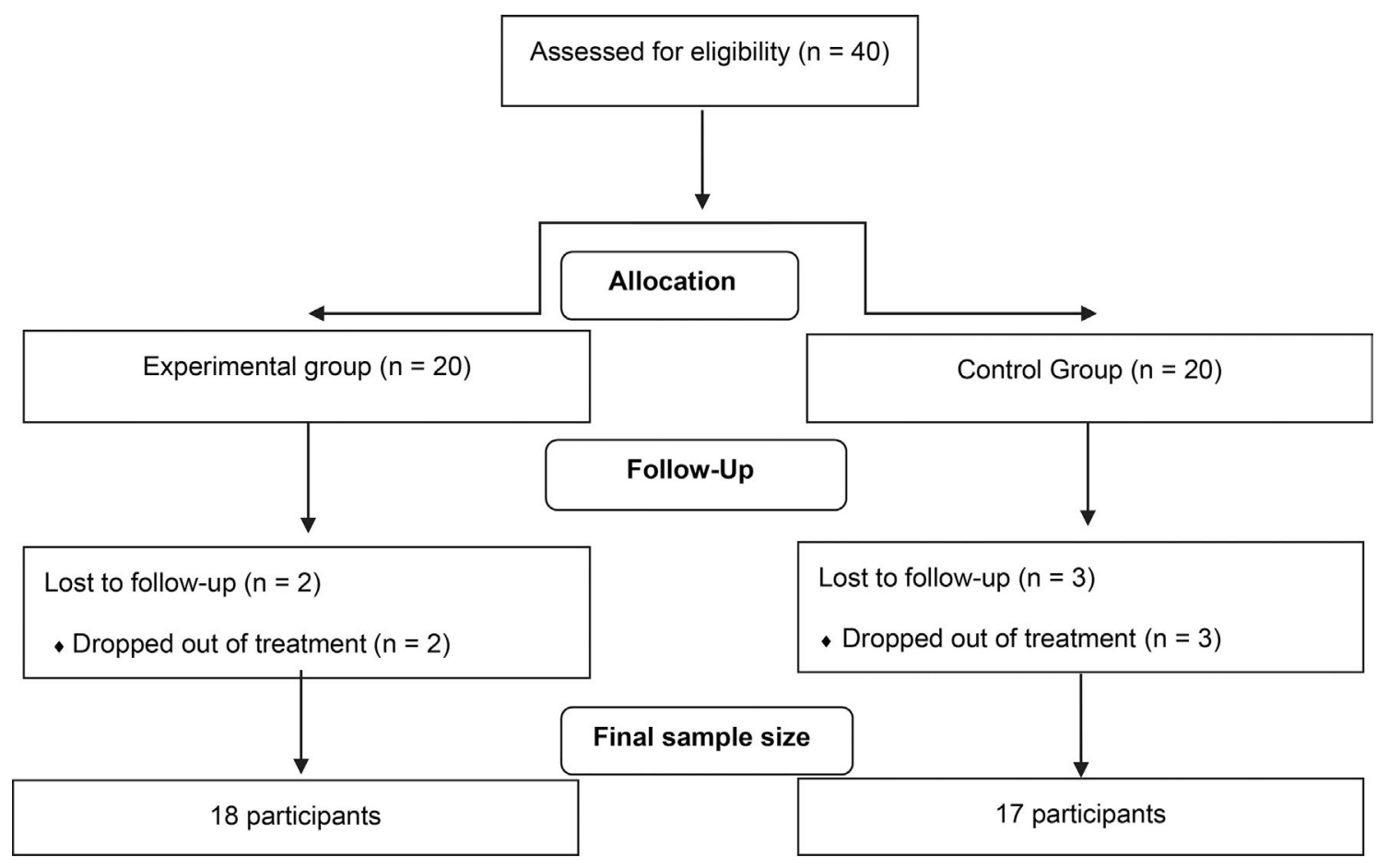

Figure 1 - Flowchart describing the study sample and distribution of participants. 
Table 1 - Participants' characteristics

\begin{tabular}{lccc}
\hline Characteristic & Experimental group & Control group & p \\
\hline Age, years, mean \pm SE & $60.33 \pm 2.23$ & $60.53 \pm 2.37$ & 0.952 \\
Time since the stroke, months, mean \pm SE & $60.00 \pm 12.91$ & $43.00 \pm 12.42$ & 0.575 \\
Right-handedness coefficient, mean \pm SE & $91.11 \pm 3.09$ & $95.00 \pm 2.84$ & $24.94 \pm 1.06$ \\
MMSE Score, mean \pm SE & $22.06 \pm 1.21$ & $58.8: 41.2$ & 0.324 \\
Sex (male, female), $\%$ & $61.1: 38.9$ & $58.8: 41.2$ & 0.083 \\
Hemisphere affected (right: left), \% & $50.0: 50.0$ & $82.4: 17.6$ & 0.890 \\
Type of stroke (ischemic, hemorrhagic), \% & $94.4: 5.6$ & $1(0-3)$ & 0.600 \\
Ashworth Scale, median (lower and higher value) & $2(1-3)$ & 0.318
\end{tabular}

Legend: SE: standard error of the mean; MMSE: Mini-Mental State Examination. Student's t-test comparing the age and MMSE score. Mann-Whitney test comparing time since the stroke, manual dominance, number of sessions, and the Ashworth Scale. Chi-square test comparing sex affected hemisphere, and type of stroke.

joints. Functional skills of the upper limbs (combing the hair, handling cutlery, holding and releasing objects, opening and closing a box, stacking blocks, and putting on a shirt) and weight transfer were also used in the exercise program.

The participants stayed three meters from the screen onto which the videos were projected. The initial posture varied depending on each movement, lying down, sitting, or standing, and ensuring a clear field of vision. The sessions occurred in groups of three to four patients. All participants had an assistant at their side who helped them achieve the range of motion requested in the exercises when needed. The participants received verbal instructions to observe the action and simultaneously imitate the movement. Verbal commands were given to direct attention and to correct the movements. Each video had fifteen repetitions of each movement.

Sessions consisted of several exercises with a oneminute rest period following each exercise. The exercises were organized by level of difficulty in each session, starting with the easiest and ending with the most difficult. The following objects were used: a $10 \mathrm{~cm}$ diameter ball, wooden sticks, drinking glasses, cutlery, a comb, a box, and a shirt. Figure 2 shows how AO was used in this study.

The control group received conventional rehabilitation, with exercises similar to the experimental group but without AO. Verbal instructions were given to perform and correct the movements requested. The sessions were conducted in groups of three to four patients, and all participants had an assistant at their side who helped them achieve the range of motion requested in the exercises, when necessary. In both groups, the frequency of treatment was twice a week and lasted approximately $40 \mathrm{mi}$ nutes, over a six-month period.

\section{Measurement battery}

Three assessments were performed: baseline, after three and six months. The variables evaluated were functional recovery, gross manual dexterity and the use of the

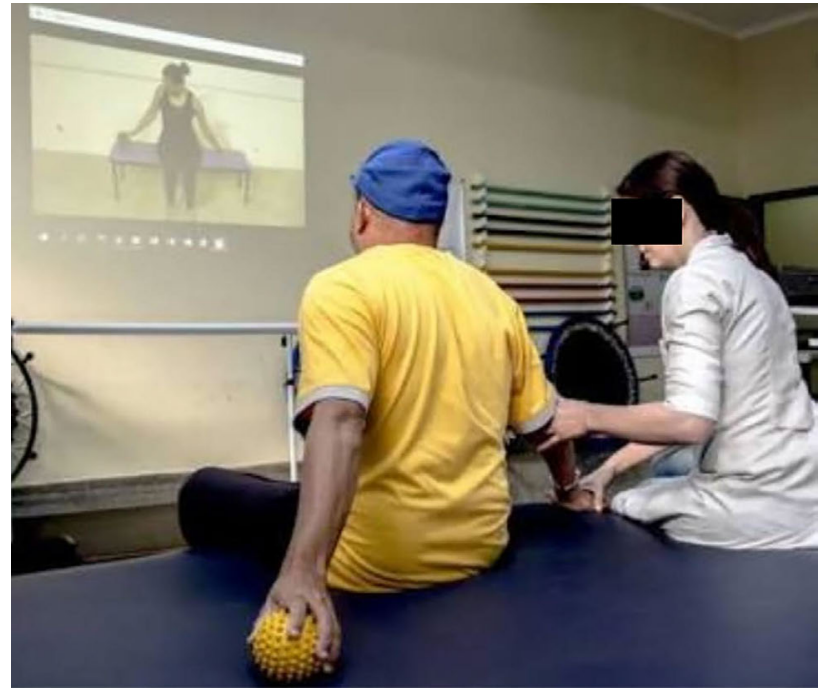

Figure 2 - Example of action observation training for participants of the experimental group.

affected upper limb. Participant was assessed individually by the same evaluator in a quiet room, seated in a chair with backrest.

The Fugl-Meyer scale ${ }^{19}$ was used to assess the functional recovery of the affected upper limb. The score varies from 0 to 66 points and evaluates mobility, speed, and coordination. The Box and Blocks Test (BBT) ${ }^{20}$ was used to evaluate gross manual dexterity. The participant moved wooden blocks from one side of a wooden box to the other side as quickly as possible. One hundred and fifty colored wooden blocks were placed in one of the box sections. Before starting the activity, the evaluator demonstrated the task and allowed 15 seconds of training for familiarization with the test. The participant performed the activity with the healthy upper limb and then with the affected one. The number of blocks moved in one minute was recorded.

The Rating of Everyday Arm-use in the Community and Home (Reach) scale ${ }^{21}$ evaluated the use of the affected upper limb. This scale is a measurement of functional 
recovery that assesses whether the participants incorporate the use of the affected upper limb in daily household and community tasks. The classification ranges from 0 to 5 , from the absence of use to total use of the affected upper limb.

\section{Statistical analysis}

Repeated measures ANOVA with the Tukey posthoc test was used for Fugl-Meyer and BBT data. The Friedman test associated with the Dunn post-hoc and Mann Whitney U tests were used for the Reach scale. The level of significance was set at $5 \%$.

\section{Results}

Results regarding the Fugl-Meyer scale, BBT and Reach scale are presented in Table 2. An improvement in the Fugl-Meyer scale was seen in both groups ( $p<0.001$, the effect size of 0.599 and power of $99.9 \%$ ), but with better results in the AO group $(\mathrm{p}<0.001)$. Both groups presented benefits in BBT scores $(\mathrm{p}=0.002$, the effect size of 0.264 and power of $95.8 \%$ ), but with similar results in between the groups $(p=0.461)$. The results of the Reach scale indicate significant improvement only in the AO group $(\mathrm{p}<0.001)$.

\section{Discussion}

This study compared the effects of an exercise program with $\mathrm{AO}$ versus conventional therapy on the function of the affected upper limb in stroke patients. The results indicated that both groups achieved significant benefits in the Fugl-Meyer scale scores and that the experimental group presented superior results. This finding corroborates previous studies ${ }^{12-17}$ that observed improvement in motor impairment of the affected upper limb after training with AO. The improvement can be attributed to the fact that exercises with $\mathrm{AO}$ stimulate mirror neurons ${ }^{22}$ and the cerebellar-thalamic-cortical system responsible for externally guided movements ${ }^{10}$.

Performing movement simultaneously with AO observation improve performance as it offers visual feedback for the correction of the movement. The characte- ristics of the exercise program may contribute to justify the results. The use of objects, videos in the third-person perspective, bimanual exercises, and verbal commands as factors that increase the excitability of primary motor were important to promote benefits to patients ${ }^{23}$.

The progression of the exercises by the level of difficulty may be another factor contributing to the improvement of patients. With repetitive training, a reduction in the excessive activation of the brain occurs in conjunction with a reduction in the cognitive effort associated with the task. This may be owing to an increase in the efficiency of synaptic transmission and contribute to functional recovery $^{17,18}$. In exercises involving bimanual coordination, the temporal or spatial characteristics of the movement path on the non-paretic side interfere with the movement path on the paretic side, improving its performance. As a result, the movements of the paretic side occur more quickly, more accurately, and more smoothly than when they are performed unilaterally ${ }^{23}$. In our study, the experimental group perceived greater use of the affected upper limb than the control group. Focusing attention with $\mathrm{AO}$ on the paretic side could contribute to the inclusion of the affected upper limb on activities of daily living and a reduction of the impact of the stroke ${ }^{14}$.

Both exercise programs improved the manual dexterity of the participants, in a similar way. This finding goes against a previous study that showed better performance of subjects stimulated with $\mathrm{AO}^{24}$. In the present study, the exercises involved multi-articular coordination of the upper limb, and not only object manipulation activities. It is known that repetitive practice of simple tasks can lead to motor improvement by adapting the short-term effects of AO to motor learning. These adaptive effects, differently, may not be present in complex motor $\operatorname{tasks}^{25,26}$. More studies are needed to address the impact of OA on complex tasks in adults with stroke.

\section{Practical applications}

This study tested an exercise program with $\mathrm{AO}$ and the results indicate that stroke patients present functional gains, which can contribute to incorporate the affected upper limb in activities of daily living. Exercises with AO

Table 2 - Comparison of treatment results in the experimental and control groups.

\begin{tabular}{|c|c|c|c|c|c|}
\hline Variable & Groups & Assessment 1 & Assessment 2 & Assessment 3 & $\mathbf{p}$ \\
\hline \multirow[t]{2}{*}{ Fulg-Meyer, score, mean \pm SE } & Experimental & $37.78 \pm 3.22^{\mathrm{a}, \mathrm{b}}$ & $41.00 \pm 3.71^{\mathrm{a}, \mathrm{c}}$ & $47.00 \pm 3.12^{\mathrm{b}, \mathrm{c}}$ & $<0.001$ \\
\hline & Control & $33.12 \pm 5.07^{\mathrm{d}, \mathrm{e}}$ & $35.18 \pm 5.14^{\mathrm{d}}$ & $36.06 \pm 5.16^{\mathrm{e}}$ & \\
\hline \multirow[t]{2}{*}{ Block and Box test, blocks/min, mean $\pm \mathrm{SE}$} & Experimental & $23.67 \pm 4.31^{\mathrm{f}}$ & $26.73 \pm 4.51$ & $29.33 \pm 4.94^{\mathrm{f}}$ & 0.002 \\
\hline & Control & $30.73 \pm 5.76$ & $31.90 \pm 5.76$ & $33.55 \pm 6.37$ & \\
\hline \multirow[t]{2}{*}{ Reach scale, ranking, median (lower and higher values) } & Experimental & $1(0-4)^{\mathrm{f}}$ & $2(0-4)$ & $3(0-5)^{\mathrm{f}}$ & $<0.001$ \\
\hline & Control & $1(0-4)$ & $2(0-4)$ & $2(0-4)$ & \\
\hline
\end{tabular}

Legend: SE: standard error. Repeated-measures ANOVA with Tukey post-test for Fugl-Meyer and Block and box test. Friedman test with Dunn post-test plus Mann Whitney-U test for the Reach scale. Superscripted letters indicate significant difference between assessments. 
may be a strategy to reinforce the learning of new tasks. In addition, the use of $\mathrm{AO}$ during rehabilitation sessions could encourage participants to do such exercises at home. However, the authors reinforce that exercises with AO do not replace conventional therapy because the findings are effective when observed tasks are corrected by professional assistance. Exercises with AO demonstrated to be a simple resource that is easily accessible by healthcare services and there were no adverse effects to treatment.

Some limitations should be pointed out, a) the sample size $(\mathrm{n}=35)$ was relatively low - even though this study contemplated the sample size calculation; b) the results are restricted to patients in the chronic phase of the stroke ( $>3$ months after stroke). Third, the researchers did not have access to the neuroimaging exams to specify precisely the brain injuries sites.

\section{Conclusion}

This study showed that AO is superior to conventional therapy in promoting benefits to patients with stroke in the following variables: functional recovery and functional use of the affected upper limb. AO presented similar results than conventional therapy on manual dexterity.

\section{References}

1. Houwink A, Nijland RH, Geurts AC, Kwakkel G. Functional recovery of the paretic upper limb after stroke: who regains hand capacity? Arch Phys Med Rehabil. 2013;94 (5):839-44. doi: 10.1016/j.apmr.2012.11.031.

2. Arwert H, Schut S, Boiten J, Vlieland TV, Meesters J. Patient-reported outcomes of hand function three years after stroke. Top Stroke Rehabil. 2018;25(1):13-9. doi: 10.1080/ 10749357.2017.1385232.

3. Lekander I, Willers C, Von Euler M, Lilja M, Sunnerhagen KS, Pessah-Rasmussen H, Borgström F. Relationship between functional disability and costs one and two years post stroke. 2017. Plos One;12(4): e0174861. doi: 10.1371/ journal.pone.0174861.

4. Connell LA, McMahon, NE, Eng JJ, Watkins CL. Prescribing upper limb exercises after stroke: A survey of current UK therapy practice. J Rehabil Med. 2014;46(3):212-8. doi: 10.2340/16501977-1268.

5. Ertelt D, Hemmelmann C, Dettmers C, Ziegler A, Binkofski F. Observation and execution of upper-limb movements as a tool for rehabilitation of motor deficits in paretic stroke patients: protocol of a randomized clinical trial. BMC Neurol. 2012:42. doi: 10.1186/1471-2377-12-42.

6. Hyeonjin J, Seung-Hwan L. From neurons to social beings: short review of the mirror neuron system research and its socio-psychological and psychiatric implications. Clin Psychopharmacol Neurosci. 2018;16(1):18-31. doi: 10.9758/ cpn.2018.16.1.18.

7. Zhang JJQ, Fong KNK, Welage N, Liu KPY. The activation of the mirror neuron system during action observation and action execution with mirror visual feedback in stroke: a systematic review. Neural Plast. 2018;2018:2321045. doi: $10.1155 / 2018 / 2321045$.

8. Hétu S, Mercier C, Eugène F, Michon PE, Jackson PL. Modulation of brain activity during action observation: influence of perspective, transitivity and meaningfulness. PLoS One. 2011;6(9):e24728. doi: 10.1371/journal. pone. 0024728 .

9. Garrison K A, Winstein C J, Aziz-Zadeh L. The mirror neuron system: a neural substrate for methods in stroke rehabilitation. Neurorehabil Neural Repair. 2010;24(5):404-12. doi: $10.1177 / 1545968309354536$.

10. Holschneider DP, Yang J, Guo Y, Maarek JM. (2007). Reorganization of functional brain maps after exercise training: importance of cerebellar-thalamic cortical pathway. Brain Research. 2007;1184:96-107. doi: 10.1016/j. brainres.2007.09.081.

11. Wright DJ, Wood G, Franklin Z C, Marshall B, Riach M, Holmes P S. Directing visual attention during action observation modulates corticospinal excitability. PLoS One. 2018;13(1):e0190165. doi: 10.1371/journal.pone.0190165.

12. Borges, LRDM, Fernandes ABGS, Melo LP, Ugerra RO, Campos TF. Action observation for upper limb rehabilitation after stroke. Cochrane Database Syst Rev. 2018;10: CD011887. doi: 10.1002/14651858.CD011887.pub2.

13. Thieme H, Morkisch N, Mehrholz J, Pohl M, Behrens J, Borgetto B, Dohle C. Mirror therapy for improving motor function after stroke. Cochrane Database Syst Rev. 2018;7: CD008449. doi: 10.1002/14651858.CD008449.pub3.

14. Sugg K, Müller S, Winstein C, Hathorn D, Dempsey A. Does action observation training with immediate physical practice improve hemiparetic upper-limb function in chronic stroke? Neurorehabil Neural Repair. 2015;29 (9):807-17. doi: 10.1177/1545968314565512.

15. Fu J, Zeng M, Shen F, Cui Y, Zhu M, Gu X, Sun Y. Effects of action observation therapy on upper extremity function, daily activities and motion evoked potential in cerebral infarction patients. Medicine (Baltimore). 2017;96(42): e8080. doi:10.1097/MD.0000000000008080.

16. Franceschini M, Ceravolo MG, Agosti M, Cavallini $P$, Bonassi S, Dall'Armi V, Massucci M, Schifini F, Sale P. Clinical relevance of action observation in upper-limb stroke rehabilitation: a possible role in the recovery of functional dexterity. A randomized clinical trial. Neurorehabil Neural Repair. 2012;26(5):456-62. doi:10.1177/ 1545968311427406.

17. Kuk EJ, Kim JM, Oh DW, Hwang HJ. Effects of action observation therapy on hand dexterity and EEG-based cortical activation patterns in patients with post-stroke hemiparesis. Top Stroke Rehabil. 2016;23(5):318-25. doi: 10.1080/10749357.2016.1157972.

18. Peng TH, Zhu JD, Chen CC, Tai RY, Lee CY, Hsieh YW. Action observation therapy for improving arm function, walking ability, and daily activity performance after stroke: a systematic review and meta-analysis. Clin Rehabil. 2019;33(8):1277-85. doi: 10.1177/0269215519839108.

19. Gladstone DJ, Danells CJ, Black SE. The Fugl-Meyer assessment of motor recovery after stroke: a critical review of its measurement properties. Neurorehabil Neural Repair. 2002;16(3):232-40. doi: 10.1177/154596802401105171. 
20. Mathiowetz V, Volland G, Kashman N, Weber K. Adult norms for the Box and Block Test of manual dexterity. Am J Occup Ther. 1985;39(6):386-91. doi: 10.5014/ajot.39.6.386.

21. Simpson LA, Eng JJ, Backman CL, Miller WC. Rating of Everyday Arm-Use in the Community and Home (REACH) scale for capturing affected arm-use after stroke: development, reliability, and validity. PLoS One. 2013;8(12): e83405. doi: 10.1371/journal.pone.0083405.

22. Kim K. Action observation for upper limb function after stroke: evidence-based review of randomized controlled trials. J Phys Ther Sci. 2015;27(10):3315-7. doi: 10.1589/ jpts.27.3315.

23. Sleimen-Malkoun R, Temprado JJ, Thefenne L, Berton E. Bimanual training in stroke: how do coupling and symmetry-breaking matter? BMC Neurol. 2011;11:11. doi: 10.1186/1471-2377-11-11.

24. Sale P, Ceravolo MG, Franceschini M. Action observation therapy in the subacute phase promotes dexterity recovery in right-hemisphere stroke patients. Biomed Res Int. 2014;2014:457538. doi: 10.1155/2014/457538.

25. Harmsen WJ, Bussmann JB, Selles RW, Hurkmans HL, Ribbers GM. A mirror therapy-based action observation protocol to improve motor learning after stroke. Neuro- rehabil Neural Repair. 2015;29(6):509-516. doi: 10.1177/ 1545968314558598.

26. Huntley MK, Muller S, Vallence AM. Corticospinal excitability is modulated by distinct movement patterns during action observation. Exp Brain Res. 2018;236(4):1067-75. doi: 10.1007/s00221-018-5199-1.

\section{Corresponding author}

Angela Cristina de Lima: Hospital Universitário da Universidade Federal da Grande Dourados, Seção de Fisioterapia. Rua Ivo Alves da Rocha 558, Altos do Indaiá. Dourados, MS, Brasil.

E-mail: angelalima@ufgd.edu.br.

Manuscript received on July 29, 2019

Manuscript accepted on February 25, 2020

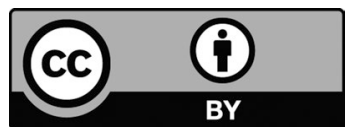

Motriz. The Journal of Physical Education. UNESP. Rio Claro, SP, Brazil - eISSN: 1980-6574 - under a license Creative Commons - Version 4.0 